Case report

\title{
Donor Derived Strongyloidiasis in a Lung Transplant Recipient: From Life Cycle to Hyperinfection Syndrome
}

Shalika B. Katugaha ${ }^{*}$, Oksana Shlobin, Christopher King, Steven Nathan, Shambhu Aryal, Kareem Ahmad, Whitney Brown

Inova Advanced Lung Disease and Transplant, VA, USA; E-Mails: skb1078@gmail.com; Oksana.Shlobin@inova.org; Christopher.King@inova.org; Steven.Nathan@inova.org; Shambhu.Aryal@inova.org; Kareem.Ahmad@inova.org; Anne.Brown@inova.org

* Correspondence: Shalika Basnayake Katugaha; E-Mail: skb1078@gmail.com

Academic Editor: Yasuhiko Sugawara

Special Issue: Current Opinion in Organ Transplantation

OBM Transplantation

2020, volume 4, issue 4

doi:10.21926/obm.transplant.2004120
Received: September 04, 2020

Accepted: October 04, 2020

Published: October 13, 2020

\begin{abstract}
Strongyloides stercoralis infection derived from the donor in solid organ transplant (SOT) places recipients at risk for hyperinfection syndrome and death. We describe the case of a lung transplant recipient who developed strongyloidiasis presenting with Gl symptoms and progressing to diffuse alveolar hemorrhage, bacteremia and multi-organ failure. The patient's clinical course illustrates the life cycle of Strongyloides. For treatment, the patient received ivermectin and albendazole. We advocate that early diagnosis and treatment, prior to hyperinfection, are essential in management in SOT. These rarer donor-derived infections need to remain in the differential, especially given the expanding landscape of the donor population.
\end{abstract}

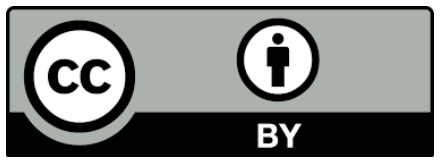

(C) 2020 by the author. This is an open access article distributed under the conditions of the Creative Commons by Attribution License, which permits unrestricted use, distribution, and reproduction in any medium or format, provided the original work is correctly cited. 


\section{Keywords}

Strongyloides stercoralis; strongyloidiasis; lung transplant; donor derived; hyperinfection syndrome

\section{Introduction}

Strongyloides stercoralis is a soil-transmitted nematode endemic to the tropics and subtropics with foci of infection occurring in temperate regions [1] where poor sanitation or other factors facilitate transmission. Immigrants and refugees comprise a significant population at risk for the helminthiasis in developed countries [2]. Acute infection commonly manifests with generalized abdominal pain, diarrhea, and emesis [3]. As these symptoms mimic other more common disease entities like viral gastroenteritis, Strongyloides infection can escape early diagnosis without a high index of suspicion [4]. In immunocompetent individuals, infection is typically self-limited and responsive to anthelmintic agents such as ivermectin. However, among immunosuppressed individuals, including the posttransplant population, lack of early detection can lead to disseminated infection and hyperinfection syndrome (HS). This may result in rapid multi-organ failure and death [5]. Despite use of anti-parasitic agents, mortality rate in disseminated disease is greater than fifty percent [6].

Cases of HS have been documented in the post-transplant population, and most have been attributed to reactivation of chronic infection in the recipient that escaped detection prior to transplant. Notably, there have been a rising number of cases of HS resulting from donor-derived (DD) infection not detected during pre-transplant screening [7, 8]. A 2012 report by the Centers for Disease Control (CDC) described seven cases of donor-derived strongyloidiasis between 2009-2012 and underscored the need to reassess the screening policies for both donors and recipients from endemic regions [9]. Current guidelines from the American Society of Transplantation recommend screening for strongyloidiasis among recipients from highly endemic regions; however, there remains no policy provision for the screening of all donors, regardless of origin [10].

We report the case of a patient who received single lung transplant and developed donor derived (DD) strongyloidiasis and HS. Given that the infection was derived from the donor, we observed manifestations of the life cycle of the parasite in her clinical course.

\section{Donor Characteristics}

The donor was a 38-year-old man who emigrated to the U.S. several years prior from Central America. He met criteria as an "increased risk donor" (CDC criteria) due to lack of available medical history, but not because of country of origin or risk for endemic disease [9]. He was serologically positive for both cytomegalovirus (CMV) and Epstein-Barr virus (EBV) and had bronchoalveolar lavage cultures positive for Stenotrophomas maltophilia and Kluyvera ascorbata. No other information or pending test results was communicated at the time of transplant. 


\section{Case Presentation}

A 40-year-old African American female with interstitial lung disease due to mixed connective tissue disease (CMV negative, EBV negative) received a left single lung transplant. She had serology negative for Strongyloides on pre-transplant evaluation. She had never traveled outside of the US. She did not receive induction therapy. Her post transplantation immunosuppressive regimen consisted of tacrolimus, mycophenolate and corticosteroids. Antimicrobial prophylaxis included voriconazole, valganciclovir and atovaquone. She had a relatively uneventful post-operative clinical course and was discharged on post-operative day (POD)\# 12 to an acute rehabilitation hospital and then subsequently home.

She presented on POD\#47 with progressive nausea, vomiting, early satiety and diarrhea over the prior 24 hours. Physical exam was notable for diffuse abdominal tenderness. She had leukocytosis with a left shift and 3\% peripheral eosinophilia (Table 1). Blood and stool cultures, C. difficile PCR and stool ova and parasites were all negative. Computed tomography (CT) of the chest, abdomen and pelvis demonstrated no focal pathology. The patient was admitted for probable gastroenteritis and started on empiric ciprofloxacin. She continued to have nausea and vomiting, and erythromycin was added for possible gastroparesis with some therapeutic benefit.

Table 1 Relevant laboratory data from date of discharge from index transplant admission to death.

\begin{tabular}{llllll}
\hline & $\begin{array}{l}\text { Discharge post- } \\
\text { transplant } \\
\text { admission, } \\
\text { POD\#12 }\end{array}$ & $\begin{array}{l}\text { Presentation, } \\
\text { POD\#47 }\end{array}$ & $\begin{array}{l}\text { Intubation, anti- } \\
\text { helminthic } \\
\text { started POD\#56 }\end{array}$ & $\begin{array}{l}\text { Refractory } \\
\text { acidemia, } \\
\text { CRRT } \\
\text { POD\#60 }\end{array}$ & $\begin{array}{l}\text { Death, } \\
\text { POD\#63 }\end{array}$ \\
\hline WBC & 8.92 & 11.83 & 5.94 & 8.91 & 3.35 \\
Neutrophils (\%) & 76 & 73 & Not done & 88 & Not done \\
Band neutrophils (\%) & 0 & 10 & Not done & 6 & Not done \\
Eosinophils (\%) & 1 & 3 & Not done & 0 & Not done \\
Platelet count & 313 & 295 & 201 & 157 & 50 \\
Creatinine (mg/dL) & 1.1 & 1.3 & 0.7 & 1.3 & 0.5 \\
\hline
\end{tabular}

By (hospital day) HD\#3/POD \#50, she developed acute respiratory failure with hypoxemia, with room air oxygen saturations in the low-80\%s while at rest. CT chest angiogram was negative for pulmonary embolism but revealed worsening left upper lobe ground glass opacities in a posterior distribution. On HD\#4/POD\#51, bronchoscopy was performed and the bronchoalveolar lavage was noted to be slightly sanguineous. By HD\#6/POD\#53, her respiratory status worsened. Antibiotics were broadened to include vancomycin and aztreonam (due to penicillin allergy), and pulsed-dose methylprednisolone was initiated for concern of graft rejection. Planned upper endoscopy for continued Gl symptoms was delayed due to clinical instability. She had progressive hypoxemic respiratory failure and on HD\#9/ POD\#56, required mechanical ventilation. Repeat CT chest revealed 
continued progression of the ground glass opacities. Bronchoscopy with lavage of the RUL was consistent with diffuse alveolar hemorrhage.

On HD\#9/POD\#56 the United Network for Organ Sharing (UNOS) communicated to us that the donor had positive Strongyloides IgG and there was concern for donor derived Strongyloides infection. The patient was immediately started on oral ivermectin $200 \mathrm{mcg} / \mathrm{kg}$ daily. The following day, she underwent upper endoscopy which showed markedly inflamed duodenal mucosa with biopsies positive for numerous parasites, morphologically consistent with invasive Strongyloides (Figure 1).

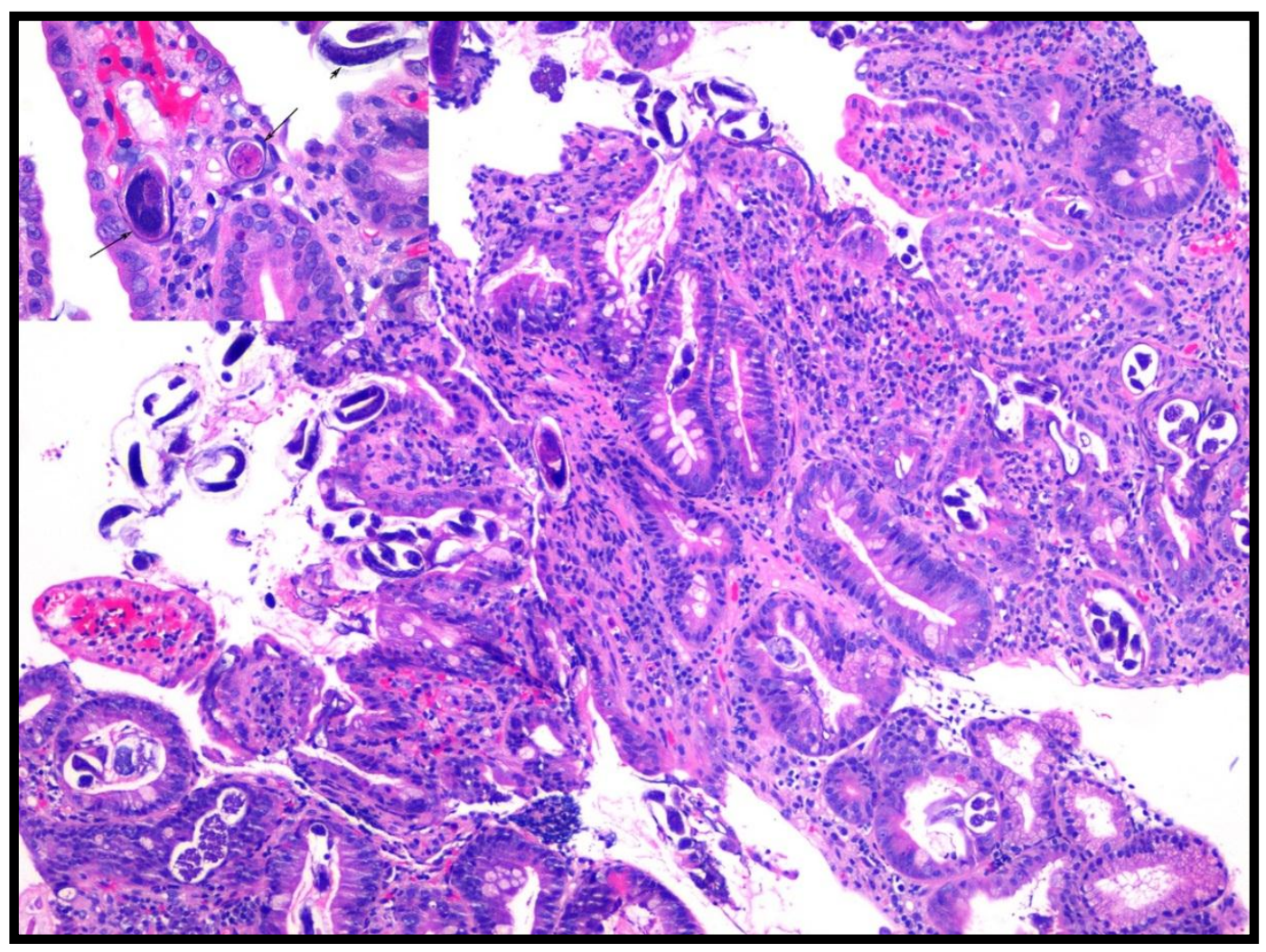

Figure 1 Duodenal biopsy revealed parasites morphologically consistent with Strongyloides both on the luminal aspect (inset, small arrow indicating adult parasite in lumen) as well as tissue invasive forms (inset, long arrows indicating larvae in lacteals) (H\&E, 100x; inset left upper corner 200x).

The recipient remained critically ill on mechanical ventilation and quickly developed multi-organ failure marked by adult respiratory distress syndrome (ARDS), acute kidney injury and shock. Blood cultures from HD\#9/POD\#56 revealed vancomycin resistant Enterococcus (VRE), consistent with a systemic sign of disseminated disease as larvae in the large intestine migrate to other organs pulling mucosal wall bacteria along with them. The patient's antibiotics were broadened to meropenem and linezolid to cover pneumonia and VRE bacteremia. On HD\#11/POD \#58, albendazole was added to the anti-parasitic regimen. On HD\#12/ POD \#59, rectal ivermectin was added, and she received intravenous immune globulin. Continuous renal replacement therapy (CRRT) for refractory acidemia was initiated on HD\#13/POD\#60. 
Due to clinical deterioration and question of absorption of the ivermectin, we sought and received Food and Drug Administration and Institutional Review Board approval for the veterinary subcutaneous formulation of ivermectin, and she was administered subcutaneous ivermectin starting on HD\#14/POD\#61. Additionally, inhaled nitric oxide and systemic paralytics were employed for refractory hypoxemia. Despite the aggressive measures, the patient did not improve and ultimately died on HD\#16/POD\#63.

\section{Discussion}

We present a case of hyperinfection syndrome as a result of donor-derived strongyloidiasis. With transmission of the parasite to a recipient on immunosuppression, we see the life cycle of the parasite play out from the Gl symptoms caused by larvae in the gastrointestinal tract to disseminated strongyloidiasis with VRE bacteremia following high dose intravenous steroids. The augmentation of immunosuppression likely hastened HS, although it is unclear if the avoidance of steroid bolus treatment or earlier initiation of anti-helminthic therapy would have afforded a different clinical outcome. Of note, the patient did not have significant eosinophilia following presentation. Corticosteroid therapy promotes eosinophil apoptosis reducing the value of this marker in the immunosuppressed host [11].

The case highlights the need for early identification of risk factors for and diagnosis of donorderived parasitic infection, which can mimic more common complications of lung transplantation. The case underscores the importance of communication between transplant centers and organ procurement organizations (OPOs). Seamless and longitudinal communication between OPOs and transplant centers is critical, particularly as the increased demand for organs has prompted the routine use of organs from higher risk donors. This is particularly salient in the setting of increasing immigration from developing countries where parasitic infections are endemic. There is a common, imperceptible mind shift from donor-derived complications to recipient-centered issues that occurs in the first week post-transplant. Ongoing recognition of the potential for longer lasting donor-derived complications would enable a more comprehensive differential diagnosis to include infections such as Strongyloides infection.

Additionally, given the continued case reports of Strongyloides hyperinfection in the transplant community, we advocate for serologic screening of all donors and recipients who have ever lived or traveled to areas endemic for strongyloidiasis. We can consider the example of Norway in the United States. Following the cases of two transplant recipients who developed Stronglyoides HS after organ transplantation from the same deceased donor in Scandinavia - the first donor-derived strongyloidiasis cases in Scandinavia, all deceased donors in Norway are screened for strongyloidiasis (not just donors originating from endemic areas) [12]. While transmission of parasitic infection from donor to recipient is a rare event, it is preventable and has potentially devastating consequences without intervention. Treatment in the early post-transplant period is, however, simple and highly effective at preventing morbidity. With the knowledge of Stronglyoides infection or prior infection in either donor or recipient, clinical suspicion and early treatment for this parasitic infection could be instituted and catastrophic outcomes potentially avoided. 


\section{Author Contributions}

The case report was initially written by Shalika Katugaha, Whitney Brown, Oksana Shlobin and Christopher King. The case was edited, and major revisions provided by Steven Nathan, Shambhu Aryal and Kareem Ahmad.

\section{Competing Interests}

None of the authors has any proprietary interests or conflicts of interest related to this submission.

\section{References}

1. Schar F, Trostdorf U, Giardina F, Khieu V, Muth S, Marti H, et al. Strongyloides stercoralis: Global distribution and risk factors. PLoS Negl Trop Dis. 2013; 7: e2288.

2. Nutman TB. Human infection with Strongyloides stercoralis and other related Strongyloides species. Parasitology. 2017; 144: 263-273.

3. Buonfrate D, Requena-Mendez A, Angheben A, Muñoz J, Gobbi F, Van Den Ende J, et al. Severe strongyloidiasis: A systematic review of case reports. BMC Infect Dis. 2013; 13: 78.

4. Agrawal V, Agarwal T, Ghoshal UC. Intestinal Strongyloidiasis: A diagnosis frequently missed in the tropics. Trans R Soc Trop Med Hyg. 2009; 103: 242-246.

5. Marcos LA, Terashima A, Dupont HL, Gotuzzo E. Strongyloides hyperinfection syndrome: An emerging global infectious disease. Trans R Soc Trop Med Hyg. 2008; 102: 314-318.

6. Roxby AC, Gottlieb GS, Limaye AP. Strongyloidiasis in transplant patients. Clin Infect Dis. 2009; 49: 1411-1423.

7. Hamilton KW, Abt PL, Rosenbach MA, Bleicher MB, Lavine MS, Mehta J, et al. Donor-derived Strongyloides stercoralis infections in renal transplant recipients. Transplantation. 2011; 91: 1019-1024.

8. Patel G, Arvelakis A, Sauter BV, Gondolesi GE, Caplivski D, Huprikar S. Strongyloides hyperinfection syndrome after intestinal transplantation. Transpl Infect Dis. 2008; 10: 137-141.

9. Hasan A, Le M, Pasko J, Ravin KA, Clauss H, Hasz R, et al. Transmission of Strongyloides stercoralis through transplantation of solid organs - Pennsylvania, 2012. MMWR Morb Mortal Wkly Rep. 2013; 62; 264-266.

10. Fischer SA, Avery RK, AST Infectious Disease Community of Practice. Screening of donor and recipient prior to solid organ transplantation. Am J Transplant. 2009; 9: S7-S18.

11. Camargo LF, Kamar N, Gotuzzo E, Wright AJ. Schistosomiasis and strongyloidiasis recommendations for solid-organ and transplant recipients and donors. Transplantation. 2018; 102: 27-34.

12. Nordheim E, Olafsson Storr $\varnothing$ M, Natvik AK, Birkeland Kro G, Midtvedt K, Varberg Reisaeter A, et al. Donor-derived strongyloidiasis after organ transplantation in Norway. Transpl Infect Dis. 2019; 21: e13008. 
OBM Transplantation 2020; 4(4), doi:10.21926/obm.transplant.2004120

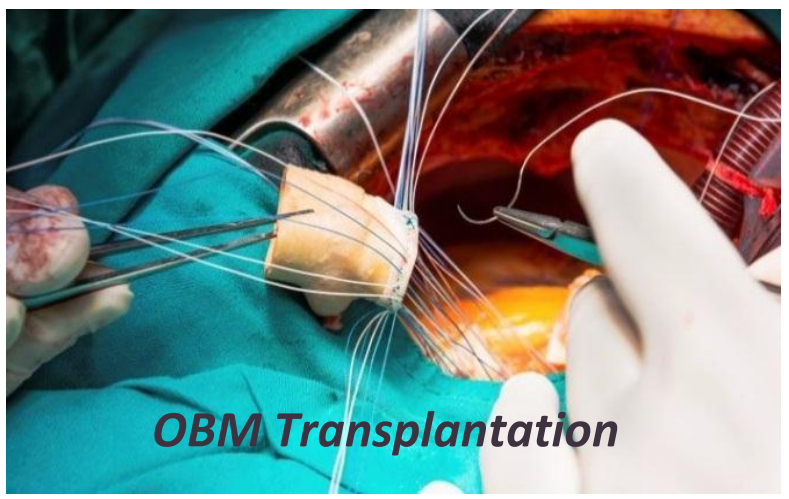

Enjoy OBM Transplantation by:

1. Submitting a manuscript

2. Joining in volunteer reviewer bank

3. Joining Editorial Board

4. Guest editing a special issue

For more details, please visit:

http://www.lidsen.com/journals/transplantation 